\title{
Les espaces publics dans les territoires de densités intermédiaires : conceptions, usages et potentialités
}

Public spaces in intermediate density areas: design logics, uses and potential

Öffentliche Räume in zwischenverdichteten Gebieten: Planungskonzepte, Nutzungspraktiken, Potenziale

Xavier Desjardins and Antoine Fleury

\section{(2) OpenEdition}

\section{Journals}

Electronic version

URL: http://journals.openedition.org/rge/5297

DOI: $10.4000 /$ rge. 5297

ISSN: 2108-6478

\section{Publisher}

Association des géographes de l'Est

Printed version

Date of publication: 30 December 2014

ISSN: 0035-3213

\section{Electronic reference}

Xavier Desjardins and Antoine Fleury, «Les espaces publics dans les territoires de densités

intermédiaires : conceptions, usages et potentialités », Revue Géographique de l'Est [Online], vol. 54 /

n³-4 | 2014, Online since 09 February 2015, connection on 08 September 2020. URL : http://

journals.openedition.org/rge/5297 ; DOI : https://doi.org/10.4000/rge.5297

This text was automatically generated on 8 September 2020

Tous droits réservés 


\title{
Les espaces publics dans les territoires de densités intermédiaires : conceptions, usages et potentialités
}

\author{
Public spaces in intermediate density areas: design logics, uses and potential \\ Öffentliche Räume in zwischenverdichteten Gebieten: Planungskonzepte, \\ Nutzungspraktiken, Potenziale
}

Xavier Desjardins and Antoine Fleury

Les investigations menées pour cet article ont été conduites dans le cadre du programme de recherche "Lieux et hauts lieux des espaces de densités intermédiaires » financé par le PUCA (2012-2014). L'article s'appuie sur une enquête réalisée dans le cadre d'un atelier professionnel du Master 2 Aménagement par quatre étudiants de l'université Paris 1 Panthéon-Sorbonne, Hugo Crespy, Coralie Hyafil, Frédéric Larose et Élise Sampoux, que nous remercions vivement.

1 Les espaces publics périurbains sont souvent mal considérés (Hamelin, Razemon, 2012). Pour certains, ils sont encore immatures, trop peu développés et " soignés » au regard $\mathrm{du}$ développement résidentiel et économique de ces territoires. Pour d'autres, ils apparaissent préfigurateurs d'une évolution redoutée donnant trop de place aux automobiles et/ou soumis à des processus de privatisation, notamment des centralités commerciales ou des rues dans les ensembles pavillonnaires. Étrangement, les espaces publics périurbains ont cependant fait l'objet de peu de recherches, malgré l'importance croissante des "études périurbaines » en France (voir la synthèse de Devisme, 2006). Quant aux modes d'habiter périurbains, ils ont été principalement étudiés à partir du "logement", comme si la sphère résidentielle structurait ces territoires davantage qu'ailleurs. Les espaces publics n'ont bien sûr pas été oubliés, mais ils ont rarement été étudiés de façon globale et systématique. Dans les années 1990, les places de villages ont donné lieu à des travaux qui attirent l'attention sur les politiques de valorisation de la centralité (Fourny et al., 1997). Les spécialistes de la 
mobilité et de son ancrage territorial ont observé les espaces publics, notamment pour comprendre les modalités de la gestion de l'automobile (Brès, 2006 ; Thébert, 2005). Les espaces commerciaux et leurs usages ont bien sûr aussi fait l'objet d'attention (Sabatier, 2006). Et plus récemment, les chercheurs se sont intéressés aux espaces non bâtis. Monique Poulot (2009) analyse ainsi la fonction des espaces ouverts dans lesquels la nature prédomine soit en raison de l'état initial (agriculture, forêts) soit par une action d'aménagement (parcs), de protection (sites classés) ou d'abandon (anciennes carrières, délaissés d'infrastructures...). Vincent Banos et Bruno Sabatier (2010) pointent quant à eux les processus croisés de publicisation et de privatisation qui caractérisent ces espaces, en donnant à voir l'incertitude du jeu social et l'invention de nouvelles règles de coexistence par leurs usagers.

2 Pour saisir la spécificité de ces espaces publics, notre posture de recherche est de pratiquer un double décalage par rapport aux approches dominantes. En premier lieu, nous nous détachons d'une lecture "péri-urbaine» de ces territoires que nous nommons "territoires de densités intermédiaires». Envisager ces derniers en considérant leur position "autour » des villes conduit en effet souvent à la détection des «manques ", qui empêchent d'en faire de "vraies villes ", et des « ajouts ", qui les éloignent irrémédiablement de la "vraie campagne ». C'est pourquoi nous prenons le parti, à la suite d'autres travaux (Bonerandi, Roth, 2007), d'appréhender ces territoires pour eux-mêmes, afin d'en analyser le fonctionnement propre. En second lieu, nous faisons un pas de côté par rapport à une lecture « urbano-centrée » des espaces publics. À la suite d'une tradition sociologique initiée par Georges Simmel et Louis Wirth, l'urbanité est définie tout à la fois par la densité et l'hétérogénéité sociale (Hannerz, 1983). Dans ce cadre, l'urbanité renvoie à l'expérience sensible de l'espace public qui implique la rencontre aléatoire d'une variété de personnes et d'objets. Comment, en partant d'une telle définition, ne pas conclure à l'urbanité moindre des lieux publics des périphéries urbaines, dans lesquels les foules sont peu présentes, les visages connus plus nombreux et l'hétérogénéité sociale souvent moins grande que dans le centre d'une métropole? Pour nous détacher de cette tradition, nous ne partirons pas d'une définition sociologique de l'espace public, mais d'une lecture géographique de la variété des espaces ouverts au public pour en comprendre le sens, les logiques de conception et les usages. Nous entendons ainsi caractériser l'ensemble des espaces dont l'accès et l'usage sont possibles gratuitement pour tous, même si leur fréquentation s'avère dans les faits plutôt ponctuelle et souvent limitée dans le temps. Un des enjeux de la recherche est bien sûr de déterminer les caractéristiques réelles de cette ouverture, au regard des contraintes d'accès ou des règles d'usage que peuvent mettre en place les gestionnaires de ces espaces et des représentations de leur accessibilité que se font les individus.

3 L'hypothèse principale qui a conduit nos investigations est celle d'un décalage entre les pratiques des habitants et les formes produites par les pouvoirs publics. D'un côté, les collectivités concentreraient leurs investissements sur les espaces emblématiques au cœur des bourgs ou villages, pour renforcer l'identification et l'attractivité de leur territoire (Fourny et al., 1997; Delbaere, 2010). D'un autre côté, les habitants inventeraient un nouveau type de relations aux espaces publics, ceux-ci pouvant être des pôles commerciaux ou des espaces «naturels » (Poulot, 2013), délaissant les rues et les places. L'enjeu est d'interroger les grilles de lecture (et d'action) des pouvoirs 
publics en termes d'espaces publics, leur capacité à intégrer les évolutions des territoires et des modes de vie dans des espaces de densités intermédiaires.

\section{Enquête dans cinq communes du Val-d'Oise}

Les cinq communes étudiées - Attainville (1800 habitants), Ecouen (7300 habitants), Ezanville (près de 9000 habitants), Le Mesnil-Aubry (un peu moins de 1000 habitants) et Moisselles (environ 1250 habitants) - sont situées à une vingtaine de kilomètres au nord de Paris (fig. 1). Si Écouen est plus bourgeoise qu'Ézanville, ces différentes communes sont marquées par une assez grande diversité sociale autour d'une classe moyenne, qui, plus qu'ailleurs, est vaste. Selon les catégories de l'INSEE, Attainville, Moisselles et Le Mesnil-Aubry sont considérées comme " périurbaines "; les communes d'Écouen et d'Ézanville appartiennent quant à elles à l'agglomération parisienne, parce que dans la continuité bâtie de Paris. Toutefois, pour l'usager du chemin de fer comme pour l'automobiliste venant de Paris, c'est avant Écouen et Ézanville que l'on observe les premières coupures d'urbanisation, par quelques champs visibles depuis le train ou une forêt traversée par la route. Écouen et Ézanville ont connu un développement résidentiel progressif à partir de l'arrivée du chemin de fer, dans les années 1870 . La croissance d'Ézanville est tardive, au début du XXe siècle, passant d'environ 300 habitants en 1901 à un peu plus de 1800 en 1906. La commune gagne près d'un millier d'habitants par décennie entre 1950 et 1990, avant de se stabiliser autour de 9000 habitants depuis. Écouen a également connu une croissance lente et relativement régulière depuis l'arrivée du chemin de fer. Depuis les années 1970, le développement de ces communes est relativement contraint par la proximité de l'aéroport Roissy Charles de Gaulle. Tout se passe comme si ces communes avaient connu toutes les étapes du développement de la banlieue parisienne, mais en ne recevant que de manière très atténuée des mouvements ailleurs très brutaux. À l'heure des grands lotissements «Loucheur " de l'entre-deux-guerres, très nombreux à Goussainville quelques kilomètres plus à l'est, ne sont ici produites que quelques centaines de maisons en meulière. À l'époque des grands ensembles des années 1950 à 1970, seules quelques barres sont construites quand elles se multiplient à Sarcelles ou à Villiers-leBel, immédiatement au sud-est de la forêt d'Ecouen. Quand se développent les villes nouvelles entre 1970 et 1990, ces deux communes n'accueillent que de petits programmes résidentiels. Prospères villages céréaliers de la Plaine de France, Moisselles, Attainville et Le Mesnil-Aubry ont connu un développement pavillonnaire à partir des années 1970. Celui-ci a été toutefois limité par la proximité de l'aéroport et la valeur agronomique importante des terres. 
Figure 1 : Localisation du secteur d'étude, autour d'Écouen-Ézanville

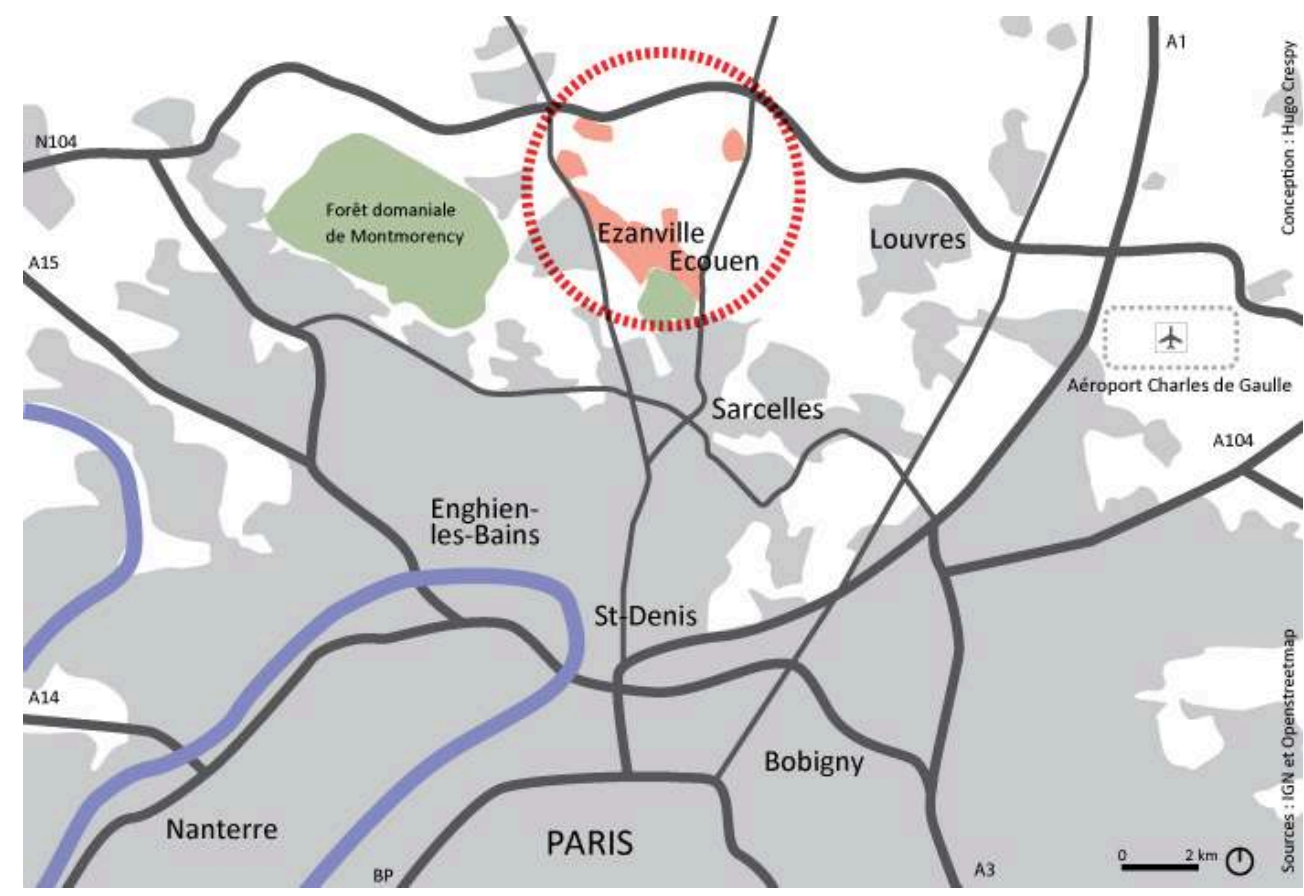

Dans chacune de ces communes ont été menées deux séries d'entretiens semi-directifs. Une première série a été menée auprès d'une vingtaine d'habitants, à leur domicile ou dans le lieu de leur choix, après une prise de contact dans des lieux publics. Pour diversifier les profils, les enquêteurs ont pris contact avec des parents d'élèves aux sorties d'écoles, avec des actifs dans les commerces ou sur le parvis des gares, avec des retraités par le biais des associations, avec les plus jeunes suite à une intervention au collège Jean-Bullant d'Écouen. Les personnes enquêtées étaient notamment invitées à commenter des photographies représentant plusieurs types d'espaces publics environnants, en évoquant leurs usages et leurs représentations de ces lieux, ce qu'ils savaient de leur fréquentation, les itinéraires empruntés dans et entre ces lieux, etc. Une autre série d'entretiens a été menée auprès d'élus et de techniciens des différentes communes du secteur d'étude, au nombre de dix. Les questions, plus classiques, avaient pour objectif de déterminer les interventions publiques sur les espaces ouverts au public considérés dans leur ensemble et de caractériser les logiques de conception. Ces deux séries d'entretiens ont été complétées par plusieurs entretiens avec des militants associatifs d'une part, des professionnels d'autre part (entrepreneurs, bureaux d'études, Architectes des bâtiments de France).

\section{Logiques de conception des espaces publics}

Les espaces publics de ces territoires sont très divers. Côté « villes ", on trouve des rues, des boulevards, des avenues, des places, des parkings, des parcs, des mails, des terrains de jeux de boule. Côté "champs" et "forêts", on trouve des chemins, des allées forestières, des promenades, des aires de cueillette. Il existe aussi des espaces nommés selon les modes technocratiques (aires de jeux, coulées vertes) ou correspondant aux évolutions de l'urbanisme commercial (les galeries commerçantes). Certains espaces n'ont pas de noms. En effet, les espaces des densités intermédiaires sont marqués par cette importance des espaces non nommés: délaissés d'infrastructure, espaces en 
attente de construction, interstices en friche. Ces espaces sont particulièrement nombreux. En effet, « une des différences les plus importantes entre la ville du passé et la ville contemporaine consiste dans les "coefficients de couverture du sol" respectifs ", nous rappelle Bernardo Secchi (2006, p. 85). Dans les espaces de densités intermédiaires, souvent appréhendés sous l'angle de la fermeture (des espaces publics) et de la densité (des constructions), c'est au contraire l'ouverture et le vide qui frappent pour l'observateur venant de la ville dense.

7 Parce que les espaces ouverts au public sont particulièrement nombreux, nous ne le présenterons pas selon leur morphologie, mais rappellerons les logiques d'action qui président à leur conception. Ces motivations sont diverses, parfois contradictoires et jamais explicites. En effet, il n'y a pas de "politique des espaces publics ». Cela existe, de manière récente, dans les plus grandes villes, même si cela reste timide: la segmentation administrative historique entre responsables des espaces verts, de la voirie, de la sécurité ou encore des transports publics se traduit souvent en rigidité intellectuelle que de nouveaux organigrammes peinent à surmonter (Fleury, 2007). Dans les communes étudiées, on ne décèle nulle trace d'une volonté d'énoncer une "politique des espaces publics». La gestion de ces derniers, tout comme leur conception, est la résultante d'actions publiques et privées multiples. On ne peut toutes les décrire, seulement en comprendre quelques-unes grâce à des entretiens auprès d'acteurs, la lecture de journaux, revues ou sites Internet tenus par des institutions, ou encore par une observation attentive de ce qui existe et de ce qui se fait (fig. 2).

Figure 2 : Projets récents et à venir pour les espaces publics du secteur d'Écouen-Ézanville

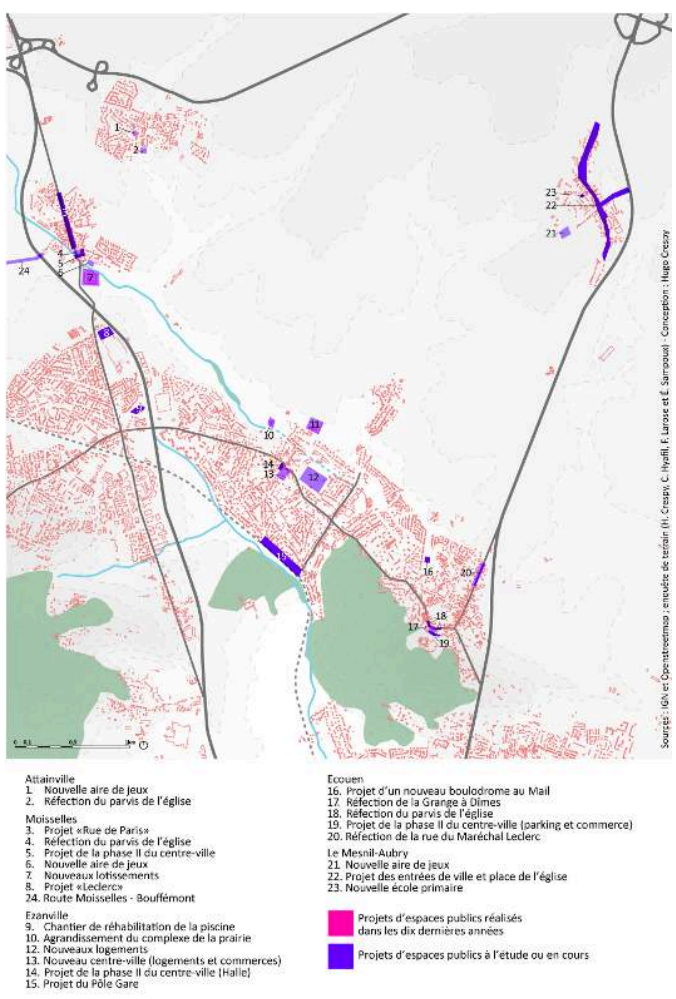




\section{A. Centralité}

Dans les cinq communes étudiées, l'attention portée aux centres anciens est tout à fait remarquable, même si elle prend des formes différentes selon la taille des communes. Ézanville a lancé dans les années 1980 un vaste réaménagement de son centre-ville. Deux édifices " centraux ", la mairie et l'église, se trouvaient loin des commerces, dans un tissu pavillonnaire assez peu structuré. C'est à mi-chemin entre les deux polarités commerciales existantes - quelques commerces près de la gare d'Écouen-Ézanville et une zone commerciale à proximité de l'échangeur de la RN 1 - que les édiles choisissent de réaliser un centre-ville. Ce projet consiste à diversifier l'offre de logements, en implantant des logements locatifs pour attirer de jeunes ménages, mais également à développer le commerce, par l'implantation de différentes boutiques dont une supérette et un café. Au cours des années 2000, la libération d'une grande parcelle occupée par des bâtiments agricoles a permis de réaliser une opération immobilière complexe en centre-ville. Un rond-point a été reconfiguré pour limiter la vitesse des automobiles, un nouveau mobilier urbain implanté. Écouen abrite depuis 1977 le musée national de la Renaissance. Perché en haut d'une butte dominant la plaine de France, le château a longtemps été coupé du reste de la ville. Lancé en 2012, le projet de la ville est de promouvoir le tourisme en s'appuyant sur quelques éléments patrimoniaux remarquables : le manoir des Tourelles, la grange à Dimes, à proximité de l'église. Le parc de stationnement situé près de l'église est réduit, au profit d'une esplanade piétonne. L'asphalte a été remplacé par des pavés, un mobilier spécifique a été choisi, potelets en acier noir et lampadaires "Vieux Paris». Un nouveau marronnier a été planté. À Moisselles, la place de l'église a été refaite. À Attainville, les alentours de la mairie sont très soignés. Si de tels aménagements n'ont pas été réalisés au MesnilAubry, une étude pour la valorisation et l'embellissement du village y a récemment été commandée; il s'agit pour elle de trouver des «solutions techniques et environnementales pour la création et l'aménagement d'espaces publics, la rénovation des voiries, la sécurisation des piétons, en adéquation avec le caractère rural de la commune $»^{1}$. Comme le souligne une élue d'Attainville, « la mode est à la rénovation des centres ", avec des ingrédients relativement similaires dans tous les cas : réduction du stationnement automobile, choix d'un mobilier «traditionnel ", mise en valeur souvent difficile - des commerces (fig. 3 et 4). L'organisation d'événements ou le soutien à des animations prises en charges par des associations locales sont l'autre volet de cette action visant à renforcer l'attractivité des centres-villes ou centresbourgs. Ainsi, quasiment toutes ont leur brocante ; le Mesnil-Aubry et Moisselles ont leur fête communale, Attainville son carnaval et Écouen son festival du Connétable, avec ses cracheurs de feu, ses vendeurs de produits locaux et ses rondes de villageois. 
Figure 3 : Attainville, son église et ses lampadaires « Vieux Paris »

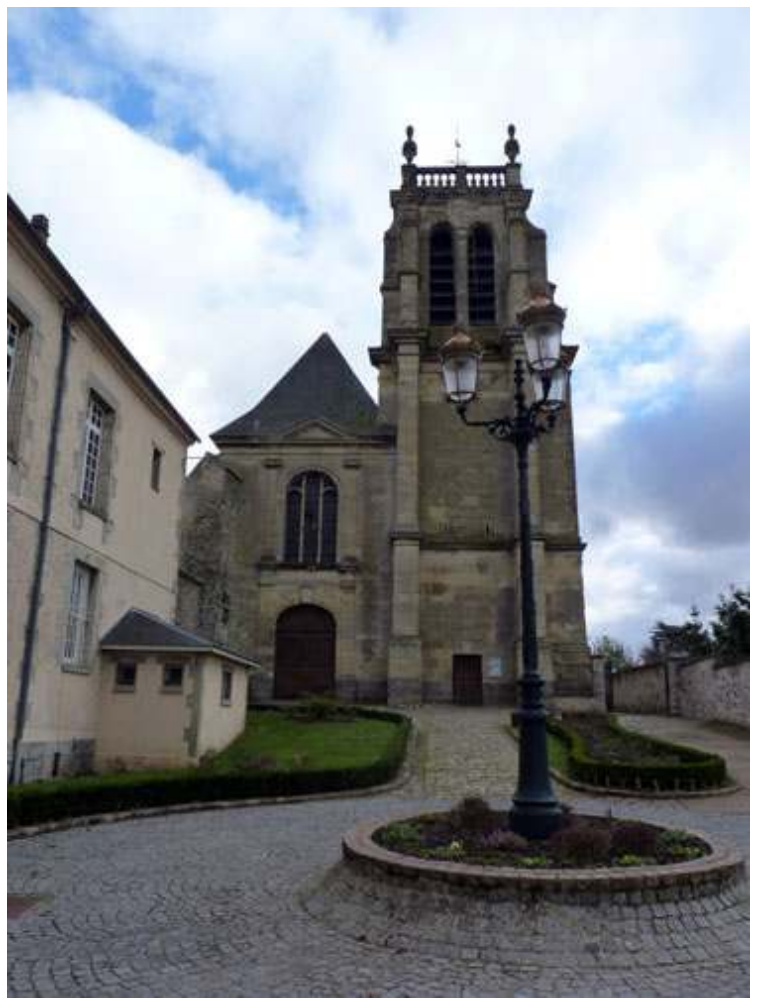

(c) Simon Labussière et Eugênia Viana, 8 février 2013

Figure 4 : Mise en valeur du patrimoine du centre ancien à Écouen

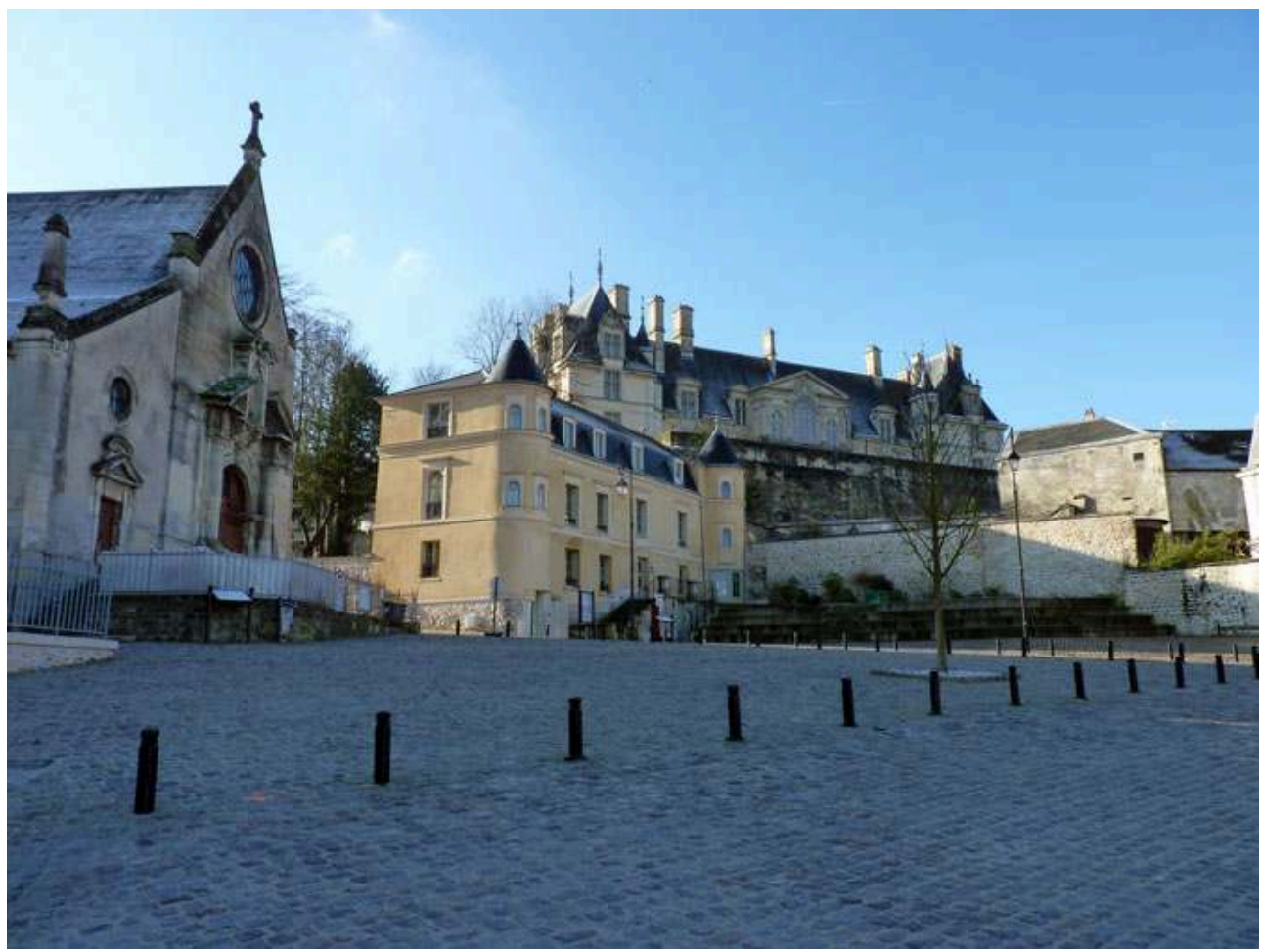

(c) Simon Labussière et Eugênia Viana, 8 février 2013 


\section{B. Circulation}

9 La gestion de la circulation demeure l'une des principales motivations dans l'évolution de la conception des espaces publics. L'espace dévolu à l'automobile est progressivement réduit. Il ne s'agit pas d'une politique contre l'usage de l'automobile : les édiles sont, dans leur grande majorité, favorables à l'amélioration des axes rapides qui forment un maillage serré dans ces franges métropolitaines. Trois routes au gabarit autoroutier ceinturent cet espace : au nord, la francilienne, à l'ouest, la RD 301 autrefois nationale 1 - et à l'est la RN 16. Il s'agit plus modestement de mieux insérer l'automobile dans les espaces habités, de se prémunir contre les excès qu'elle engendre. Il faut tout d'abord détourner le trafic de transit. Attainville, à la sortie d'une bretelle de l'A 104, use de tous les artifices pour ralentir la circulation : resserrement des voies, zone 30, circulations alternées des deux sens de circulation, dos d'âne, etc. La stratégie adoptée est similaire à celles des "quartiers verts » ou "tranquilles" des grandes agglomérations: le trafic est repoussé vers les grands axes. C'est la stratégie «Disneyland» (Joly, Crozet, 2006): des espaces de circulation très ralentie au cœur d'axes rapides (fig. 5). Toutefois, quelques signes d'un changement d'appréhension de l'automobile sont à l'œuvre. Le stationnement est un problème important près de la gare d'Écouen-Ézanville. Une étude pour le réaménagement de la gare a été lancée en 2012 avec le Syndicat des transports d'île-de-France (STIF). Quelques commerces profitent de ce lieu de passage : un café, un restaurant japonais, un kebab, un pressing, une auto-école. Mais le tout est assez peu lisible et peu animé. Parce que la gare est à la frontière entre les deux communes d'Écouen et d'Ézanville, éloignée des centres-villes, la création d'une véritable centralité autour de la gare n'est pas recherchée. Les élus veulent néanmoins un réaménagement pour rendre ce lieu plus agréable et lisible, et si possible, réduire la place de l'automobile par le développement d'axes cyclables. Les trottoirs sont élargis pour les personnes à mobilité réduite. L'éclairage de routes départementales est mis en place pour rendre plus agréable le passage de cyclistes ou de piétons à la nuit tombée (fig. 6). Ce mouvement de prise en compte des "déplacements actifs » est manifeste, notamment pour tenir compte du vieillissement de la population et de pratiques sportives massives de la course à pied ou à vélo, qui exigent des continuités de parcours. Mais ce mouvement apparaît encore bien incomplet, au regard du nombre de trottoirs impraticables ou interrompus, de la difficulté à faire du vélo. Rien n'exprime mieux cette incomplète prise en compte que les grands équipements qui ne sont pensés que pour un accès par les transports motorisés. C'est le cas des centres commerciaux bien sûr. Mais il ne faudrait pas en faire les seuls représentants de ce que l'on peut appeler des "isolats » au sein de ces territoires: le très récent collège d'Ézanville est bien représentatif de cet oubli du piéton. Le collège tourne le dos à la rue et les seuls espaces sécurisés pour les piétons conduisent depuis la sortie du collège vers les arrêts d'autobus. 
Figure 5 : Requalification et réduction de la place de l'automobile dans le centre de Moisselles

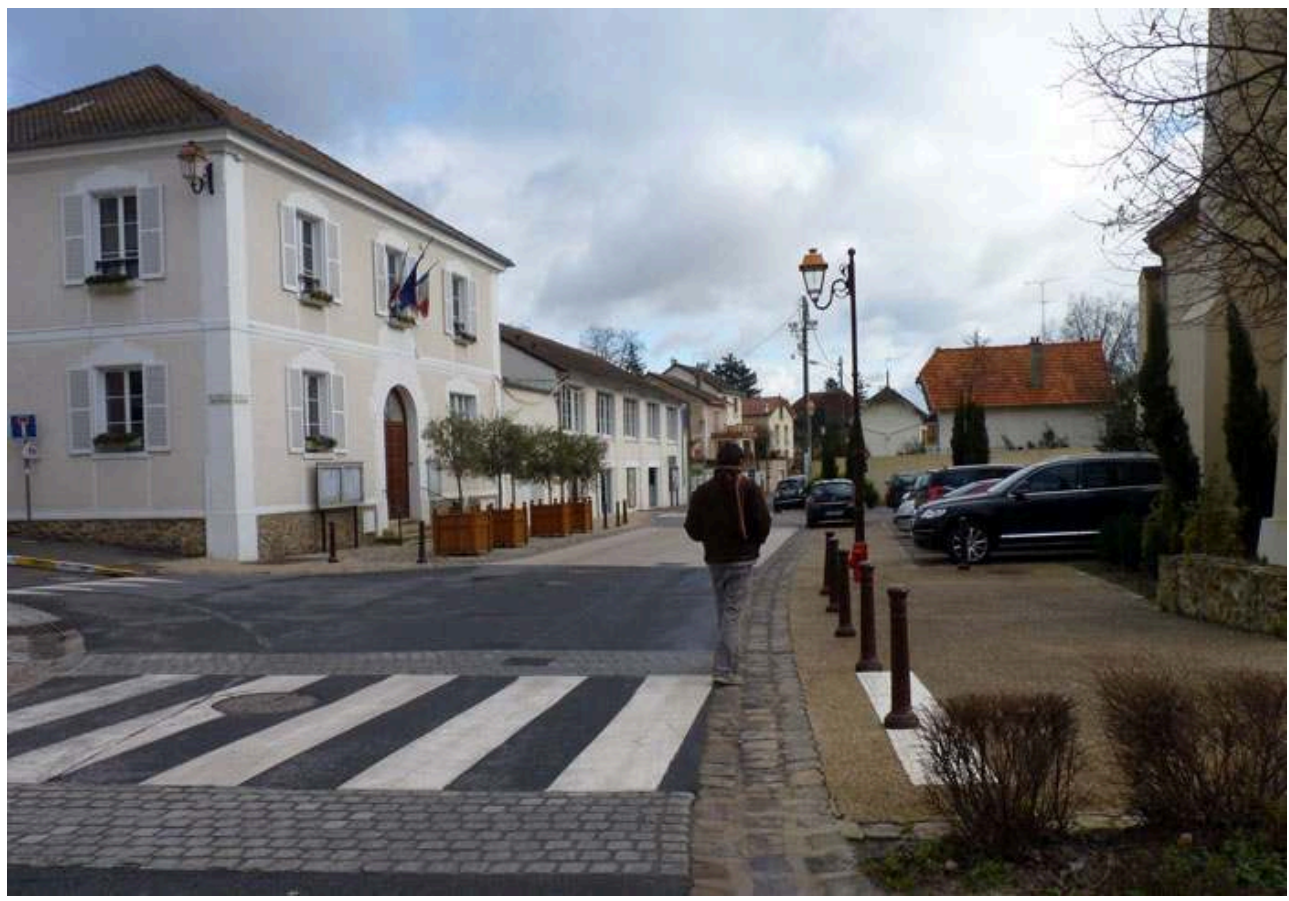

(c) Eugênia Viana, 8 février 2013

Figure 6 : Depuis Moisselles vers Attainville, une route éclairée pour les circulations piétonnières et cyclistes

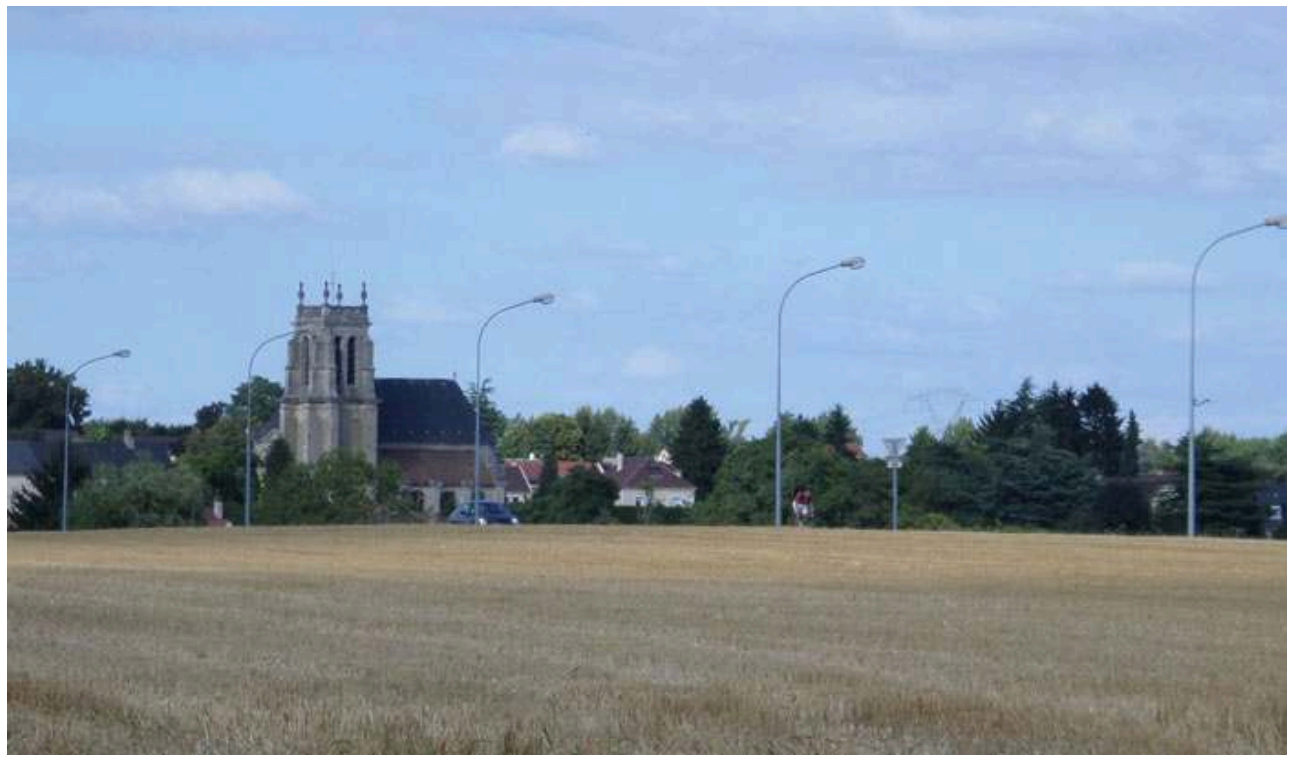

(c) Xavier Desjardins, 20 août 2013

\section{Sécurité et tranquillité}

Les enjeux de sécurité et de tranquillité sont, au-delà de la seule question de l'automobile et de ses risques, l'une des composantes importantes de la conception des espaces publics dans les territoires de densités intermédiaires. Deux types d'espaces 
publics sont concernés. Il s'agit d'une part de ceux qui posent un problème de compatibilité entre certains usages et les attentes des riverains. Ces derniers «n'apprécient pas la présence d'espace public sous leur fenêtre » nous dit un agent municipal de Moisselles, l'espace public renvoyant ce faisant à la présence prolongée de groupes d'individus, souvent jeunes, consommant parfois de l'alcool, et faisant du bruit. " Ça fait du bruit les bancs, du moment qu'on met un lampadaire et un banc ensemble, ça génère des regroupements " reconnaît la responsable du service d'urbanisme de l'une des petites communes d'étude. Il s'agit d'autre part d'espaces publics qui paraissent peu sûrs en raison de leur faible fréquentation. La faible densité et le caractère principalement résidentiel de ces communes conduit à une très faible densité d'usages des espaces publics et à une concentration dans le temps de ces usages (sorties d'écoles, promenade de fin de semaine, etc.). C'est d'autant plus le cas pour les espaces publics caractérisés par leur étendue, qui sont nombreux dans ces territoires : espaces verts périphériques et forêts principalement.

11 Les élus font donc le choix de supprimer ou de ne pas installer des bancs ou des assises dans certains espaces publics proches des habitations, notamment dans les centresvilles ou centres-bourgs. Face à l'ampleur des espaces à contrôler, c'est moins une gestion des mobilités dangereuses et des immobilités non désirées que recherchent les élus (Landauer, 2009) qu'une mise à l'écart ou un éloignement de différents usages non désirés. En même temps, les élus sont attentifs à l'éclairage public, censé apporter un sentiment de sécurité, condition essentielle à la fréquentation des espaces publics. Ainsi, à Écouen, un soin tout particulier est apporté à l'implantation des lampadaires dans des zones qualifiées de stratégiques, comme les sentes du centre-ville et des zones pavillonnaires, afin que ces espaces publics soient davantage utilisés. Il en va de même pour Ézanville où la mairie procède au remplacement des lampadaires, souvent en nombre insuffisant. Afin de sécuriser certains espaces publics sans réaliser de dépenses excessives, la mairie a même imaginé de refaire l'éclairage avec un système de luminosité variable en fonction de la fréquentation des lieux. Enfin, un certain nombre de communes se sont dotées de caméras de vidéosurveillance, en particulier autour des gares, des mairies et des entrées de ville.

\section{L'action publique à l'épreuve des usages}

Même si elle confirme l'inégale fréquentation des espaces publics, avec notamment de fortes variations dans le temps, notre enquête auprès des habitants a permis de mettre en lumière la grande variété des usages de ces espaces autant que des représentations que véhiculent ces derniers. Nous ne décrirons pas ici toute la diversité de ces espaces, de leurs usages et de leurs représentations, pour nous concentrer sur la manière dont ces usages et représentations sont pris en compte par les pouvoirs publics locaux.

\section{A. Ambivalences}

13 De manière générale, l'observation in situ a permis de constater que les centres sont relativement peu fréquentés, ce que confirment les entretiens avec les habitants: autour de l'église d'Ézanville, « on ne voit pas beaucoup de monde » et «quand il y a quelqu'un, on se demande ce qu'il fait » fait remarquer Colette. Une grande majorité des habitants interviewés ne s'y rend que très rarement, pour des achats ponctuels, 
pour des formalités administratives en mairie et lors d'événements exceptionnels. La disparition progressive des commerces - les derniers étant surtout considérés comme des points de ravitaillement de dépannage - et la faible fréquentation des églises ou des mairies expliquent en partie cette situation. Seuls les quelques événements évoqués cidessus permettent de rassembler la population dans les rues de centre-ville ou de centre-bourg. Ces événements animent de manière temporaire des centres délaissés le reste de l'année. Leur succès est le signe que les habitants, même s'ils les fréquentent rarement, valorisent beaucoup les centres-bourgs, avec pour certains d'entre eux un attachement fort. Leur dimension symbolique et identitaire est soulignée de manière récurrente :

"C'est devant la mairie que je ressens toute l'histoire de la commune et tous les bons moments vécus » (Robert ${ }^{2}$, Écouen)

«Si je suis attaché à cette image de l'église, c'est parce qu'elle évoque les racines de ce village » (Claude, Le Mesnil-Aubry).

La préoccupation des habitants à la fois pour l'image de leur commune et pour leur cadre de vie passe aussi par une grande importance attribuée à l'entretien voire, pour certains d'entre eux, à la mise en valeur de ces éléments emblématiques que sont les rues, places et monuments historiques des centres-bourgs. Au total, si l'on peut observer un décalage important, souligné par certains auteurs (Delbaere, 2010), entre le degré d'investissement des collectivités locales pour l'amélioration de ces centres et leur fréquentation réelle, l'action publique n'en rejoint pas moins très largement la représentation que se font les habitants de ces espaces. Les événements exceptionnels organisés par les mairies ou par des associations locales (avec le soutien des mairies), répondent également aux attentes des habitants pour des rassemblements dont le caractère identitaire est, encore une fois, souvent très marqué.

Les gares et les quartiers qui les jouxtent ont un statut très différent. Devant la gare d'Écouen-Ézanville, il y a certes des commerces de proximité qui amènent les habitants à y rester un peu plus longtemps quand ils y passent ou à s'y rendre ponctuellement sans prendre le train (fleuriste, pressing, tabac en particulier). Mais bien que le temps d'attente soit souvent long, les gares et leurs abords n'apparaissent pas comme des lieux de sociabilité et de rencontre :

«Il n'y a pas grand-chose » (Vanessa et Jérôme, Ézanville)

«Ce n'est pas pour autant un lieu de rencontre. C'est surtout un lieu de passage »

(Nicolas, Moisselles)

« Je fréquente ce lieu pour l'usage qu'il m'offre » (Anne, Écouen)

Les habitants qui utilisent les transports en commun n'attribuent de surcroît aucune valeur ou aucun attachement particulier à des lieux qui leur apparaissent comme essentiellement fonctionnels. Au regard de la représentation qu'ils se font des abords de gare, les habitants rejoignent donc les pouvoirs publics dans leur choix de ne pas en faire des lieux plus centraux et de privilégier une organisation plus fonctionnelle. Cependant, le choix de favoriser les déplacements "actifs " paraît en contradiction avec les usages. Ainsi, plusieurs enquêtés se plaignent du parking de la gare d'ÉcouenÉzanville qui, même s'il a été agrandi, est toujours complet et désormais payant :

«C'est chiant car maintenant le parking est zone bleue » (Ouardia, Ézanville)

"C'est toujours difficile de se garer» (Sarah, Claudine et Colette, Attainville) de se rendre à la gare en voiture et a pour principale préoccupation le stationnement. 


\section{B. Décalages}

L'enquête auprès des habitants a permis de confirmer que les champs et les forêts occupent une place importante dans l'espace de vie de ces derniers, dans la proximité de leur lieu de résidence. Ainsi, la forêt d'Écouen - en relation ou non avec le château est fréquentée régulièrement par les habitants d'Écouen et Ézanville. C'est un lieu de détente : pique-nique, repos et lecture, course à pied ou vélo. C'est aussi un lieu de passage quotidien entre la gare et le centre d'Écouen. Dans les plus petites communes et dans les lotissements périphériques d'Ézanville, ce sont les champs et chemins ruraux qui assurent ces fonctions. Certes les entretiens, complétés par l'observation in situ, confirment que la fréquentation de ces espaces ruraux est plutôt lâche, d'autant qu'elle est soumise aux aléas climatiques. Mais de nombreux enquêtés y vont régulièrement marcher, seuls ou en famille, promener leur chien, faire du jogging ou du vélo. Plusieurs enquêtés, le plus souvent des retraités, y pratiquent également la randonnée dans le cadre d'associations. Si les chemins ruraux et forestiers n'ont pas vocation à être des lieux de rencontre mais plutôt à permettre un contact avec la nature et la contemplation du paysage, ils peuvent aussi donner lieu à des rencontres fortuites :

«Je rencontre parfois des dames qui marchent. L'abord était facile car il y avait les petits chiens » (Colette, Ézanville).

«Je cours dans les champs. C'est assez connu dans le coin. Les gens promènent leur chien. On croise pas mal de gens » (Vanessa, Ézanville)

Néanmoins, ces espaces ne font pas l'objet d'attentions particulières de la part des collectivités locales. Si c'est sans doute lié au statut de certains d'entre eux, qui demeurent privés, force est de constater que les collectivités concentrent leurs investissements sur les centres au détriment de ces espaces publics dont le rôle dans la vie des habitants est pourtant considérable. On note néanmoins quelques évolutions, comme à Moisselles où une route de campagne permettant de relier le centre et la gare est désormais éclairée, à Écouen où le chemin reliant le centre-ville à la gare à travers la campagne est lui aussi éclairé, ou dans certaines communes qui ont goudronné des chemins ruraux fortement empruntés par les habitants.

L'autre grand décalage constaté entre l'action publique et les usages concerne les centres commerciaux. Nous avons pu constater, lors des phases d'observation in situ, que la galerie commerciale du Leclerc de Moisselles est un espace très fréquenté, quelle que soit l'heure de la journée. Contrairement à la plupart des espaces publics évoqués ci-dessus, elle attire une population qui dépasse à la fois la commune et le périmètre de l'enquête. Sa centralité est donc sans commune mesure avec celle des centres-bourgs évoqués précédemment. Le Leclerc de Moisselles constitue à la fois un lieu de ravitaillement et un lieu de culture, avec son « Espace culturel » très souvent cité. Pour de nombreux habitants interviewés, sa galerie commerciale apparait aussi comme un lieu de rencontre et de flânerie :

«Le Leclerc est un centre de rendez-vous pour les lycéens de Domont et les amis du rugby. Il y a un café. C'est assez vivant » (Jérôme, Ézanville)

«Quand j'ai vraiment besoin de voir autre chose, je vais au Leclerc» (Ouardia, Ézanville)

«On rencontre souvent des gens (...) Quand on veut se détendre, flâner, on va faire la galerie, quand on ne veut pas acheter » (Sylvie, Attainville) 
21 En cela, au-delà de son statut juridique, le centre commercial constitue bien un espace public à part entière (Sabatier, 2006). Loin des discours dépréciatifs que l'on peut lire sous la plume de certains journalistes ou chercheurs, il est aussi clairement valorisé par nombre d'habitants, presque autant, même si c'est sur un autre registre, que les espaces « naturels ». Pourtant, les collectivités semblent largement se désintéresser des centres commerciaux. Les cheminements piétons sont difficiles sur leurs pourtours, y compris avec les lotissements alentours, et leur intégration paysagère reste médiocre. Là encore, l'action publique apparaît comme en décalage avec les pratiques habitantes. Il en va de même pour les représentations des élus, les collectivités continuant à chercher la revitalisation commerciale des centres-bourgs. Bien plus, il faut souligner l'attitude paradoxale des élus qui à la fois cherchent à attirer sur leur territoire ces nouvelles centralités commerciales qu'ils rejettent en périphérie tout en regrettant la perte de la fonction centrale des anciens noyaux villageois.

\section{Convergences}

22 Plusieurs enquêtés évoquent des problèmes de sécurité, principalement en forêt. Pour certains, leur pratique de la forêt est même limitée par un sentiment d'insécurité : plusieurs femmes précisent n'y aller qu'accompagnées et éviter de la parcourir une fois la nuit tombée. Mais ce qui ressort le plus souvent des entretiens, ce sont les conflits d'usages déjà évoqués ci-dessus et dont les élus ont bien conscience. Un exemple parmi d'autres est le mail d'Ecouen, qui cristallise des conflits d'usages entre des familles avec enfants ou des personnes âgées qui cherchent la tranquillité et des jeunes qui ont élu résidence dans ce mail, peut être car aucun autre endroit ne se prête à leurs rendezvous. En revanche, ce qu'il y a de commun à tous les enquêtés, c'est l'attachement au calme et à la tranquillité :

«Avant, je détestais ce calme. Maintenant, c'est ce que je recherche» (Ouardia,

Ézanville)

«C'est la campagne, c'est calme » (Anne-Marie, Écouen)

La préservation du cadre de vie en tant qu'il est caractérisé par le calme et la tranquillité constitue même l'une des préoccupations majeures des habitants. Ainsi, alors que la participation aux réunions publiques concernant le projet de réaménagement du centre-ville a été extrêmement faible, le responsable des services techniques d'écouen constate que "la réunion publique sur les nuisances sonores est l'une des rares réunions où les habitants se sont mobilisés ». La présidente de l'association pour la sauvegarde de l'église d'Attainville considère même que les habitants sont plus motivés pour la protection de leur cadre de vie que pour la protection du patrimoine bâti, ce dont le succès de l'association Attainville, ma campagne témoigne. En arbitrant en faveur des riverains dans les conflits d'usages qui caractérisent un certain nombre d'espaces publics (par exemple par la suppression de bancs, comme nous l'avons vu précédemment) ou en améliorant l'éclairage public le long de certaines sentes ou rues, les pouvoirs publics de ces communes répondent donc largement à l'attente des habitants - du moins de la majorité des habitants - qui sont aussi leurs électeurs. Ce faisant, certains groupes minoritaires aux usages considérés comme déviants, en particulier les jeunes, sont cependant mis à l'écart. Spatialement tout d'abord puisque c'est en périphérie des espaces habités, dans les espaces verts, dans certains champs et forêts, qu'ils se trouvent repoussés, des espaces qui par ailleurs font l'objet d'une action publique très réduite, donnant parfois l'impression d'un 
certain abandon de ces espaces publics. Temporellement ensuite puisque leurs pratiques sont essentiellement nocturnes.

\section{Conclusion : quels espaces publics pour les espaces de densités intermédiaires?}

L'intérêt porté par les collectivités locales aux espaces publics des centres-bourgs apparaît légitime dans la mesure où ceux-ci ont une fonction symbolique importante pour les habitants. Comme le faisaient justement remarquer Fourny et al. (1997, p. 94), « en donnant sens à l'entité locale, [ce type d'intervention] en affirme l'autonomie, la distinction et la valeur par rapport aux entités plus larges de l'agglomération ou de la région urbaine ". Même s'ils ne jouent plus que de manière éphémère leur fonction de rassemblement, ces espaces n'en sont pas moins un repère et un élément de cohésion pour les habitants et les acteurs du territoire, qui justifie en partie les investissements réalisés. Les aménagements mis en œuvre dans les centres-bourgs doivent-ils pour autant demeurer une priorité de l'action publique? Au regard des pratiques et des représentations des habitants, on ne peut que s'étonner du faible intérêt porté aux centres commerciaux d'une part, aux espaces "naturels » d'autre part, même si les choses commencent à changer. L'essentiel de l'action publique consiste le plus souvent encore à appliquer les mêmes recettes que dans la ville dense, sans que soient réellement prises en compte les spécificités de ces territoires et des modes de vie qui s'y déploient.

C'est donc plus globalement la conception des espaces publics qui pose problème. Elle demeure marquée par une lecture par «points " - " entrées de villes », ronds-points, places, espaces verts, etc. - plus que par une lecture par «lignes » et par " réseaux ». Même si cette deuxième lecture se retrouve pour l'aménagement des axes, ceux-ci sont avant tout traités comme des axes de circulation automobile. Cette tendance à privilégier les «points » sur les « lignes " renvoie finalement à une vision réductrice des déplacements et des usages associés aux espaces publics, qui vont des pratiques de proximité, à pied ou à vélo, aux déplacements à plus longue distance, en automobile. Depuis quelques années, l'attention aux pratiques de proximité et aux cheminements piétons est plus importante. Mais ce souci est assez incomplet, au regard des nombreux axes routiers et de la conception des équipements qui les bordent. Les interventions demeurent très localisées, négligeant le "potentiel de proximité » des espaces de densités intermédiaires dans leur ensemble. Or il faut accepter de changer de regard sur ces espaces en les considérant comme « un agrégat de situations urbaines distinctes mais reliées de façon fluide et ouverte » (Delbaere, 2010, p. 75). L'enjeu est de mieux prendre en compte - voire de promouvoir - des pratiques de proximité généralisées entre les logements, les commerces, les équipements publics, les gares et les espaces « naturels ».

Il faut pour cela concevoir les espaces publics selon la logique de la trame, mais en sortant des modèles de la ville dense pour mieux prendre en compte le niveau intermédiaire qui caractérise ces espaces. Il faut inventer des aménagements qui soient favorables aux piétons et aux cyclistes ainsi qu'à la multiplicité des usages, mais qui soient en même temps adaptés à une densité modeste de la fréquentation. Quand il est apporté un soin particulier à un espace ouvert au public, par exemple dans les centresbourgs, on retrouve souvent les mêmes matériaux, les mêmes mobiliers que dans la 
ville dense (potelets, lampadaires, revêtement du sol, etc.). Pour les trames dédiées aux piétons et vélos dans les espaces de densités intermédiaires, ne faut-il pas inventer un modèle à coût réduit? L'enjeu des espaces publics des densités intermédiaires n'est-il pas de trouver un juste milieu entre le non-aménagement et un luxe parfois surprenant? Récemment, le paysagiste V. Delbaere proposait que l'aménagement des espaces publics se fasse de manière "négociée (avec la géographie, les usages économiques, avec les tracés produits par les usages préexistants) et ferme (par la simplicité, la rigueur et l'extrême visibilité de son dispositif)", "sur la base d'investissements modestes»(2010, p.110). Nous le rejoignons sur ce point, en insistant davantage cependant sur l'importance de la prise en compte de tous les usages et de tous les espaces dans lesquels ils s'insèrent, que ce soit les espaces "naturels ", appelés à devenir de plus en plus structurants (Vanier, 2011), les centres commerciaux et les gares.

\section{BIBLIOGRAPHY}

Banos V., Sabatier B., 2010, « Les espaces périurbains non bâtis en France : entre publicisation "urbaine" et privatisation "rurale" ?", Articulo - Journal of Urban Research, http:// articulo.revues.org/1524.

Brès A., 2006, « De la voirie à la rue : riveraineté et attrition. Des stratégies d'inscription territoriale des mobilités périurbaines ", Flux, nº66-67, pp. 87-95.

Delbaere D., 2010, La Fabrique de l'espace public. Ville, paysage et démocratie, Paris, Ellipses, 186 p.

Devisme L. (dir.), 2006, La structuration du périurbain, synthèse bibliographique, PUCA, DRE Pays de la Loire, 116 p.

Fleury A., 2007, Les espaces publics dans les politiques métropolitaines. Réflexions au croisement de trois expériences : de Paris aux quartiers centraux de Berlin et Istanbul, Thèse de doctorat, Université Paris 1 Panthéon-Sorbonne, $685 \mathrm{p}$.

Fourny M.-C., Pagand B., Pradeilles J.-C., 1997, « Les nouveaux centres péri-urbains : l'espace public porteur de territoires », Revue de géographie alpine, Tome 85, $\mathrm{n}^{\circ}$ 4, pp. 83-95.

Hamelin E., Razemon O., 2012, La tentation du bitume, où s'arrêtera l'étalement urbain ?, Rue de l'échiquier, $225 \mathrm{p}$.

Hannerz U., 1983, Explorer la ville, traduit et présenté par Isaac Joseph, Paris, Editions de Minuit, Coll. Le sens commun, 418 p.

Crozet Y., Joly I., 2006, La « Loi de Zahavi » : quelle pertinence pour comprendre la construction et la dilatation des espaces-temps de la ville?, Plan Urbanisme Construction Architecture (PUCA), Coll. Recherches du PUCA, $n^{\circ} 163,89 \mathrm{p}$.

Landauer P., 2009, L'architecte, la ville et la sécurité, PUF, Coll. « La ville en débat », 101 p.

Poulot M., 2013, « Du vert dans le périurbain », EspacesTemps.net, http://www.espacestemps.net/ articles/du-vert-dans-le-periurbain-les-espaces-ouverts-une-hybridation-de-lespace-public/. 
Sabatier B., 2006, La publicisation des espaces de consommation privés. Les complexes commerciaux récréatifs en France et au Mexique, Thèse de doctorat, Université de Toulouse II Le Mirail, 476 p.

Secchi B., Première leçon d'urbanisme, Marseille, Éd. Parenthèses, 155 p.

Thébert M., 2007, «Stratégies communales et gestion des déplacements : les maires du périurbain rennais face à la mobilité », in Mattéi M.-F., Pumain D. (dir.), Données urbaines 5, Paris, Économica, pp. 29-36.

Vanier M., 2011, « La périurbanisation comme projet », Métropolitiques, http:// www.metropolitiques.eu/La-periurbanisation-comme-projet.html

\section{NOTES}

1. Source: site Internet de la commune, consulté le 6 décembre 2013, URL: http://www.lemesnil-aubry.fr/environnement/urbanisme/.

2. Tous les prénoms ont été modifiés pour garantir l'anonymat des personnes enquêtées.

\section{ABSTRACTS}

Public spaces in periurban areas often have a poor image. They are considered either as insufficiently developed, or as emblematic of privatised town development. To overcome this simplistic vision, and in order to understand the specificity of these areas, this article seeks to distance itself both from a "periurban" reading of these areas and from an "urban-centred" view of public spaces. Based on a survey conducted in the northern outskirts of the Paris region, it highlights the design logic of public authorities, as well as the convergences and discrepancies with the inhabitants' behaviour. Finally it puts forward some possibilities for action.

Souvent perçus négativement, les espaces publics périurbains sont considérés soit comme insuffisamment développés, soit comme emblématiques de la ville privatisée. Pour sortir de cette vision réductrice et en saisir la spécificité, cet article entend prendre ses distances par rapport à une lecture "péri-urbaine " de ces territoires et à une lecture "urbano-centrée " des espaces publics. À partir d'une enquête menée sur les franges nord de l'agglomération parisienne, elle met en évidence les logiques de conception véhiculées par les pouvoirs publics, les convergences et les décalages avec les pratiques des habitants, avant de proposer quelques pistes pour l'action.

Öffentliche Räume am Rande von Städten leiden gewöhnlich an einem Imagedefizit: Entweder werden sie als nicht zahlreich genug dargestellt oder sie gelten als symptomatisch für die Stadtprivatisierung. Im vorliegenden Artikel soll diese vereinfachende Sichtweise hinterfragt und die Spezifität dieser Gebiete in Betracht gezogen werden. Suburbane Gebiete sind eben keine "Randgebiete"; genauso wenig können suburbane öffentliche Räume mit stadtzentrierten Kategorien analysiert werden. Anhand einer nördlich des Pariser Ballungsraums geführten qualitativen Untersuchung werden hier die spezifischen Planungslogiken der öffentlichen Hand gezeigt, sowie deren Konvergenzen bzw. Diskrepanzen mit Bewohnerpraktiken. Zum Schluss werden einige operative Vorschläge gemacht. 
INDEX

Mots-clés: densités intermédiaires, espaces publics, politiques publiques, usages

Schlüsselwörter: lokale Raumordnungspolitik, Nutzungspraktiken, Öffentliche Räume, Zwischenverdichtung

Keywords: intermediate densities, public policies, public spaces, uses

\section{AUTHORS}

\section{XAVIER DESJARDINS}

Université Paris 1 Panthéon-Sorbonne - UMR Géographie-cités (CNRS, Universités Paris 1

Panthéon-Sorbonne et Paris Diderot - Paris 7) - xavier.desjardins@univ-paris1.fr

\section{ANTOINE FLEURY}

CNRS - UMR Géographie-cités (CNRS, Universités Paris 1 Panthéon-Sorbonne et Paris Diderot Paris 7) - afleury@parisgeo.cnrs.fr 